\title{
Fluorescência óptica no diagnóstico de lesões em colo cervical - revisão de literatura
}

\author{
Optical fluorescence in the diagnosis of cervical colosion injuries - literature review \\ Fluorescencia óptica en el diagnóstico de lesiones en colo cervical - revisión de literatura \\ Gabriela Garutti PEREIRA ${ }^{1}$ \\ Jessica Gielize Fernandes da SILVA ${ }^{1}$ \\ Daiane Fernanda Pereira Mastrocola BIZELLI ${ }^{2}$ \\ Luciana Estevam SIMONATO ${ }^{2}$ \\ ${ }^{1}$ Graduandas do Curso de Biomedicina da Fundação Educacional de Fernandópolis, 15600-000 Fernandópolis - SP, Brasil \\ ${ }^{2}$ Docentes do Curso de Biomedicina da Fundação Educacional de Fernandópolis, 15600-000 Fernandópolis - SP, Brasil
}

\section{Resumo}

O câncer de colo uterino ainda hoje é um problema de saúde pública em muitas partes do mundo. No Brasil, é o terceiro tumor maligno mais frequente entre as mulheres. Uma das causas pontadas como percussor desse câncer é o HPV, principalmente, quando correlacionado a outros fatores de riscos e estilo de vida. Sua cura é altamente possível desde que se tenha um diagnóstico rápido e preciso. Na maioria dos casos, as alterações celulares no colo uterino são descobertas facilmente no exame preventivo conhecido como Papanicolau. Outro método que pode ser utilizado para esta detecção precoce de lesões em colo cervical é a fluorescência óptica que não é invasivo e vem sendo amplamente utilizado no diagnóstico de lesões bucais e de pele. Dessa forma, o presente trabalho teve como objetivo realizar uma revisão da literatura sobre a aplicação da fluorescência óptica no diagnóstico de lesões em colo cervical.

Descritores: Fluorescência; Neoplasias do Colo do Útero; Diagnóstico.

\section{Abstract}

Cervical cancer is still a public health problem in many parts of the world today. In Brazil, it is the third most frequent malignant tumor among women. One of the leading causes of this cancer is HPV, especially when correlated with other risk factors and lifestyle. Its cure is highly possible as long as you have a quick and accurate diagnosis. In most cases, cellular changes in the cervix are easily discovered in the preventive examination known as Papanicolaou. Another method that can be used for this early detection of cervical lesions is non-invasive optical fluorescence and has been widely used in the diagnosis of oral and skin lesions. Thus, the present work had the objective of reviewing the literature on the application of optical fluorescence in the diagnosis of cervical lesions.

Descriptors: Fluorescence; Uterine Cervical Neoplasm; Diagnosis.

\section{Resumen}

El cáncer de cuello uterino sigue siendo un problema de salud pública en muchas partes del mundo. En Brasil, es el tercer tumor maligno más frecuente entre las mujeres. Una de las causas planteadas como percusión de este cáncer es el VPH, principalmente, cuando está correlacionado con otros factores de riesgo y estilo de vida. Su curación es altamente posible desde que se tenga un diagnóstico rápido y preciso. En la mayoría de los casos, los cambios celulares en el cuello uterino se descubren fácilmente en el examen preventivo conocido como Papanicolaou. Otro método que puede ser utilizado para esta detección precoz de lesiones en cuello cervical es la fluorescencia óptica que no es invasiva y viene siendo ampliamente utilizado en el diagnóstico de lesiones bucales y de piel. De esta forma, el presente trabajo tuvo como objetivo realizar una revisión de la literatura sobre la aplicación de la fluorescencia óptica en el diagnóstico de lesiones en cuello cervical.

Descriptores: Fluorescencia; Neoplasias del Cuello Uterino; Diagnóstico.

\section{INTRODUÇÃO}

Para o Brasil, as estimativas para 2018, produzidas pela Divisão de Vigilância e Análise de Situação da Coordenação de Prevenção e Vigilância do Instituto Nacional de Câncer (INCA) ${ }^{1}$, são de 600 mil novos casos de câncer. À exceção do câncer de pele não melanoma, os tipos de câncer mais incidentes em homens serão próstata $(31,7 \%)$, pulmão $(8,7 \%)$, intestino $(8,1 \%)$, estômago $(6,3 \%)$ e cavidade oral $(5,2 \%)$. Nas mulheres, os cânceres de mama $(29,5 \%)$, intestino $(9,4 \%)$, colo do útero $(8,1 \%)$, pulmão $(6,2 \%)$ e tireoide $(4,0 \%)$ figurarão entre os principais ${ }^{2}$.

O câncer de colo do útero ocupa o terceiro lugar em incidência nas mulheres e possui diversos fatores de risco relacionados a sua etiologia, tais como: uso prolongado de anticoncepcionais, iniciação sexual precoce, alta paridade, tabagismo, infecção por clamídia, entre outros. No entanto, o principal causador desta doença é a infecção persistente pelo Papilomavírus Humano (HPV) ${ }^{2-4}$

Muitas mulheres, principalmente as de baixa renda, com pouco acesso à educação e à saúde, tem dificuldade para obter um diagnóstico precoce ${ }^{4,5}$. No entanto, a prevenção do câncer cervical é altamente possível, uma vez que pode ser realizada por meio de programas de rastreamento, tratamento de lesões precursoras e tratamento do $\mathrm{HPV}^{6,7}$.

Atualmente, os principais meios de diagnóstico consistem na realização de exames ginecológicos, exames de citologia oncótica, colposcopia e biópsia- ${ }^{7-9}$. Esses exames possuem um tempo para liberação dos seus resultados, o que pode gerar estresse e ansiedade para a paciente ${ }^{10}$. Logo, o desenvolvimento de novas tecnologias para a detecção do câncer de colo uterino se faz necessário, para que o diagnóstico seja precoce. Especialmente, se esse método permitir a liberação do diagnóstico de imediato.

De encontro com essa necessidade, pode-se lançar mão de métodos já existentes e verificar sua aplicabilidade em novas situações, como por exemplo, a fluorescência óptica que, atualmente, é utilizada com grande frequência na detecção de lesões bucais e de pele.

Sendo assim, o objetivo do presente teve como objetivo realizar uma revisão da literatura sobre a aplicação da fluorescência óptica no diagnóstico de lesões em colo cervical. 


\section{REVISÃO DE LITERATURA}

O câncer de colo do útero ocupa a terceira colocação em incidência, mas apresenta um alto índice de prevenção e cura. Isso acontece porque, por meio dos exames preventivos, é possível detectar as lesões pré-malignas e, ainda, graduar as lesões neoplásicas intraepiteliais cervicais (NIC). A graduação dessas lesões precursoras são: NIC I, NIC II e NIC III ${ }^{1,2}$.

A NIC I é considerada uma lesão de baixo grau de malignidade e possui displasia leve, sendo que na maioria dos casos há regressão. Já a NIC II e a NIC III são consideradas lesões com displasia moderada e grave, que exigem acompanhamento e tratamento ${ }^{1,11}$.

Quando essas lesões precursoras progridem e chegam ao tecido conjuntivo são chamadas de carcinoma invasor, não sendo obrigatório passar pelos três estágios de NIC $^{1}$.

Como citado anteriormente, o principal fator considerado como precursor é a infecção pelo HPV. Cabe ressaltar que isoladamente o vírus não é capaz de induzir a mudança de uma célula saudável para uma célula neoplásica, mas associado a outros fatores de riscos, como: tabagismo, iniciação da vida sexual precoce e vários parceiros, pode haver evolução. Atualmente, existem vacinas para os tipos 6, 11, 16, 18 do HPV, sendo que os tipos 16 e 18 são os que apresentam maior risco para o desenvolvimento dos carcinomas invasores ${ }^{3,4,11}$.

Quanto mais precoce for o diagnóstico do câncer de colo do útero, maiores são as chances de cura. O tratamento é realizado de acordo com o estadiamento clínico da lesão, a idade da mulher, a condição sistêmica da paciente e o desejo ou não de ter filhos ${ }^{2}$.

$\mathrm{O}$ exame de Papanicolaou é considerado um método de fácil aplicabilidade e de baixo custo, que possibilita o seu uso no sistema público de saúde, atingindo assim um grande número de mulheres. Além de ser o exame de eleição para os programas de rastreamento do câncer de colo do útero ${ }^{1}$.

Esse exame consiste na análise microscópica das células esfoliadas da cérvice, com a finalidade de detectar anormalidades celulares. As células são coletadas por meio de um método simples que tem início com a inserção do espéculo na vagina, viabilizando que o médico realize a visualização direta do colo do útero. Em seguida, com uma espátula de Ayre e uma escovinha endocervical o profissional provoca uma escamação da superfície e as células colhidas são colocadas em uma lâmina histológica para fixação, coloração e análise $e^{4,10}$.

A citologia oncótica não é de aplicação exclusiva para câncer de colo do útero, ela também é utilizada para a detecção de outras lesões em diferentes locais do corpo, como por exemplo o câncer de pele ${ }^{12}$.
Outro método de uso rotineiro é a colposcopia, que é um método de análise visual dos tecidos e vasos sanguíneos do colo do útero, que utiliza um microscópio de filtro verde de baixa potência para a visualização de possíveis lesões na região, facilitando assim a biópsia e o planejamento do tratamento adequado. A técnica consiste no posicionamento de um espéculo seguido de colocação de solução salina para limpeza e ácido acético a $4 \%$ para desidratação do tecido, permitindo identificar onde há proliferação de células anormais ${ }^{13}$.

Em caso de detecção de anormalidades, tanto no exame de Papanicolau quanto na colposcopia, há indicação de realização de biópsia, a qual consiste na retirada de um pedaço de tecido para análise microscópica da morfologia celular ${ }^{10}$.

Por indicação do Ministério da Saúde, a biópsia não é indicada como a primeira escolha no diagnóstico das lesões precursoras do câncer uterino, pois é invasiva e requer maior habilidade técnica ${ }^{7}$.

O desenvolvimento de tecnologias ópticas oferece dados importantes sobre a morfologia e a constituição bioquímica das células, o que faz deste método uma ferramenta eficiente e bastante promissora para a análise patológica e diferenciação de tecidos normais e lesados, favorecendo um diagnóstico precoce ${ }^{14,15}$.

Um sistema de fluorescência óptica muito utilizado é o EVINCE (MMOptics, São Carlos, SP, Brasil), que é composto por LEDs de alta potência, que emitem desde o espectro eletromagnético até a radiação ultravioleta, variando o comprimento de onda de $380 \mathrm{~nm}$ a $450 \mathrm{~nm}$, além de um conjunto de filtros ópticos que permitem a visualização da área visualizada $^{16,17}$.

Quando as células recebem o feixe de luz, ocorre uma excitação a nível molecular, ocorrendo trocas de níveis de energia. Nesta troca, a célula dispersa essa energia para o ambiente. Alguns fluóforos, como: colágeno, elastina, NADH e FAD permitem a visualização quando a energia é dispersada ${ }^{13}$.

Atualmente, este método é muito aplicado na área odontológica para observar os duros dentais e em tecidos moles para detecção de lesões potencialmente malignizáveis. Outra aplicação, também, muito utilizada é para a detecção de lesões malignas em câncer de pele ${ }^{14,16,17}$. O colo do útero é revestido por epitélio escamoso estratificado não queratinizado à semelhança da mucosa oral. Dessa forma, a ampliação de estudos com fluorescência óptica no colo cervical é fortalecida pelos bons resultados verificados nos estudos em cavidade oral.

O contraste da luz nos tecidos com lesões precursoras ou neoplásicas, depende da profundidade das lesões, sendo este um fator importante para a análise da espectroscopia. Com a correta distribuição 
das fibras ópticas para excitarem as células é possível uma análise correta ${ }^{13,18}$.

A espectroscopia da fluorescência óptica oferece informações que permitem a verificação de diferenciações bioquímicas nas células sem a necessidade de fluóforos exógenos e, também, possibilita a observação das características estruturais dos tecidos em suas condições de normalidade ou patológica, sendo possível a observação in vivo ${ }^{18}$.

É possível verificar, ainda, que a proporção $\mathrm{NADH} /$ colágeno-elastina em tecido patológico está aumentada em relação ao tecido normal. Isto ocorre, pois em estados patológicos há variações de metabolismo, distribuição ao longo do tecido ou estruturas alteradas que afetam as taxas de espectro de fluorescência ${ }^{13}$.

\section{CONSIDERAÇÕES FINAIS}

Ao revisar a literatura em relação aos métodos de diagnóstico para o câncer cervical, percebe-se que os métodos utilizados como rotina possuem um tempo de espera para a liberação do laudo. Dessa forma, mesmo a fluorescência óptica não sendo um método de diagnóstico utilizado para as lesões em colo cervical atualmente, seria possível viabilizar um diagnóstico precoce, sem ser invasivo e sem o estresse da espera para a paciente com o seu uso, assim como já é realizado na detecção de lesões bucais e na pele.

\section{REFERÊNCIAS}

1. INCA. Instituto Nacional de Câncer. Coordenação de Prevenção e Vigilância (Conprev) Falando sobre câncer do colo do útero. Rio de Janeiro: MS/INCA, 2002.

2. Brasil. Ministério da Saúde. Secretaria de Atenção à Saúde. Instituto Nacional de Câncer. Coordenação de Prevenção e Vigilância. Estimativa 2018: incidência de câncer no Brasil. Rio de Janeiro: INCA; 2017.

3. WHO. World Health Organization. Human papillomavirus (HPV) and cervical cancer. 2016. Disponível em: $<\mathrm{http}: / / \quad$ www.who.int/ mediacentre/factsheets/fs380/en/>. Acesso em: 02 set. 2017.

4. Martins LTF, Fraga CDS, Andrade MS, Santos KJS, Paixão GPN, Bittencourt IS. Caracterização de mulheres com lesão pré-maligna ou maligna no exame papanicolaou. Rev Enferm UFPE.2017; 11(9):3360-68.

5. Moreira TR, Lima ACS, Santos MA, Auler ME, Turkiewicz M, Chaves MAF, Plewka J. Perfil das mulheres usuárias do SUS com lesões intraepiteliais em um município do oeste do Paraná. Arq Ciênc Saúde UNIPAR. 2017;21(3):181-86.

6. Guarisi R, Hardy E, Derchain SFM, FonsechiCarvasan GA, Borges JBR. Rastreamento diagnóstico e tratamento das lesões precursoras e do câncer invasor de colo uterino no município de Franco da Rocha, SP. Rev bras cancerol. 2004;50(1):7-15

7. Lima TM, Lessa PR, Freitas LV, Teles LMR, Aquino OS, Damasceno AKC et al. Análise da capacidade diagnóstica dos exames preventivos do câncer de colo uterino. Acta paul enferm. 2012; 25(5):673-78.

8. Brasil. Ministério da Saúde. Conduta INCA/MS Câncer do colo do útero. Rev bras cancerol. 2000; 46(4):351-54.

9. Trindade GB, Manenti AS, Simões PW, Madeira K. Avaliação do rastreamento do câncer do colo do útero e sua periodicidade em um município de Santa Catarina. Medicina. 2017;50(1):1-10.

10.Acosta DF, Dantas TS, Cazeiro CC, Acosta DF, Gomes VLO. Vivenciando o exame papanicolau: entre o (não) querer e o fazer. Rev enferm UFPE. 2017; 11(8):3031-38.

11.Derchain SFM, Longatto Filho A, Syrjanen KJ. Neoplasia intraepitelial cervical: diagnóstico e tratamento. Rev Bras Ginecol Obstet. 2005;27(7):425-33.

12.Lins B, Sartor BC, Scariot PK, Tusset C. Citologia oncótica: aplicabilidade e atuação do profissional biomédico na área. In: Congresso de Pesquisa e Extensão da Faculdade da Serra Gaúcha. Caxias do Sul. 2014;318-27.

13. Giraldo BS. Espectroscopia óptica de fluorescência aplicada ao soporte de diagnóstico médico de précanceres de tejidos de cuello uterino [tese]. Manizales: Universidad Nacional de Colombia. Fac Ingenieria y Arquitetura; 2009.

14.Coelho VHM. Fluorescência óptica no diagnóstico de lesões teciduais [tese]. São Carlos: Universidade de São Paulo. Instituto de Química de São Carlos; 2005.

15. Yassoyama MCBM. Estudo do colo uterino por Espectroscopia FT-Raman [dissertação]. São José dos Campos: Universidade do Vale do Paraíba; 2006.

16. Ricci HA, Pratavieira S, Brugnera Junior A, Bagnato VS, Kurachi C. Ampliando a visão bucal com fluorescência óptica. Rev Assoc Paul Cir Dent. 2013;67(2):129-35.

17. Simonato LE, Tomo S, Miyahara GI, Navarro RS, Villaverde AGJB. Fluorescence visualization efficacy for detecting oral lesions more prone to be dysplastic and potenttially malignant disorders: a pilot study. Photodiagnosis Photodyn Ther. 2017; 17(1):1-4. 
18.Corti MA, Garavalia MJ. Biopsia optica. Descrición general y resultados preliminares por espectroscopia optica e autoflorescencia. Externos. 2015;1(1):707-10.

\section{CONFLITO DE INTERESSES}

Os autores declaram não haver conflitos de interesse.

\section{AUTOR PARA CORRESPONDENCIA}

Luciana Estevam Simonato

luciana.simonato@universidadebrasil.edu.br

Submetido em 29/05/2018

Aceito em 27/06/2018 